\title{
Hangover Syndrome: Pathogenesis and Treatment
}

\section{Razvodovsky YE* (D)}

Department of Medico-Biological Problems of Alcoholism, Institute Biochemistry of Biologically Active Substances Academy of Science of Belarus, Russia

*Corresponding author: Razvodovsky YE, Head of the Department of Medico-Biological Problems of Alcoholism, Institute of Biochemistry of Biologically Active Compounds, National Academy of Sciences of Belarus, Belarus, 230009, Grodno, 50 BLK str., Russia

\begin{abstract}
Aim: To discuss the state of art of pathogenesis and treatment of Hangover Syndrome (HS)

Methods: The review of the Russian and English language literature related to this problem.

Results: It was shown, that there is a significant gap in the understanding of pathogenesis of HS, which hamper the elaboration of means of metabolic correction of this condition. The target of majority of currently existing means of treatment of HS is one of the pathogenic mechanisms of HS. The effectiveness of majority of currently existing medicine is not supported by the results of clinical trials.

Conclusions: The actual task of future research is the detailed investigation of pathogenic mechanisms of HS. This will allow elaborating the comprehensive medicine for HS treatment targeting all links of its pathogenesis.
\end{abstract}

\section{Keywords}

Hangover syndrome, Pathogenesis, Treatment

\section{Introduction}

Alcohol abuse causes significant damage to health and is associated with more than two hundred different diseases [1]. One of the most common negative consequences of alcohol abuse is the so-called post-intoxication, or hangover syndrome, which is a symptom complex of psychophysiological disorders that develop the morning after an alcoholic excess (heavy alcohol consumption) the night before [2-9]. The clinical symptomatology of HS varies greatly. In the classical form, HS manifests itself as a constellation of physiological (general malaise, headache, fatigue, nausea, dry mouth, thirst, lack of appetite) and mental (low mood, impaired concentration, cognitive deficit) symptoms $[5,10]$.

A study on volunteers who injected themselves with alcohol intravenously showed that the morning after self-administration of alcohol $78 \%$ of the participants experienced at least one hangover symptom [6]. The most common symptoms of HS were: Fatigue (67\%), thirst $(57 \%)$, and headache (32\%) [6]. The symptoms of a HS appear 6-8 hours after the end of alcoholic excess and can be observed for 20 hours against the background of the absence of alcohol in the blood [10]. The existing gender and age specificity of $\mathrm{HS}$ is in the greater severity of symptoms in women [5], as well as its progression with increasing age [3].

Hangover is associated with a host of medical and socioeconomic problems. HS can pose a threat to health, as it provokes an increase in blood pressure and heart rhythm disturbances, which, in turn, can cause sudden death [11]. HS also carries significant socioeconomic implications. For example, in the United States, the annual economic losses due to decreased productivity associated with hangover syndrome are about $\$ 179$ million [5].

In experimental studies, it was shown that in the state of $\mathrm{HS}$, the reaction time and the number of errors in the task performance increase [12,13]. Impairment of cognitive functions (attention, operative, retrospective and prospective memory, deficit of executive function) and psychomotor disorders noted in HS lead to a decrease in labor productivity increase the risk of industrial and road traffic injuries [14-16]. There is a direct relationship between the frequency of hangover and the likelihood of various kinds of problems in the workplace 
[3]. A simulator study showed a significant impairment in the ability to drive in a state of hangover [17]. Meanwhile, $56.4 \%$ of heavy truck drivers in Denmark admitted driving in a state of hangover [6]. A biochemical marker survey of drivers admitted to a US hospital with injuries following a road traffic accident showed that $22 \%$ of them drank alcohol on the eve of the accident, although no alcohol was found in their blood [5].

Since HS is associated with alcoholic excesses, it is considered a sign of alcohol abuse [4]. This is confirmed by the fact that the severity of HS is positively correlated with the level of alcohol problems, assessed using the AUDIT test [5]. Symptoms of HS can resemble the clinical picture of mild withdrawal symptoms, which is one of the key diagnostic criteria for alcohol dependence [3]. However, unlike HS, withdrawal symptoms are manifested by more pronounced and varied symptoms (including neurological symptoms), which manifests itself against the background of a strong craving for alcohol [6]. Drinking alcohol greatly alleviates the symptoms of withdrawal symptoms [14]. HS is characterized by a lack of craving for alcohol, while an aversion to the smell of alcohol may appear which allows us to consider HS as a post-intoxication state [18]. Nevertheless, despite the differences in the clinical picture and pathogenic mechanisms, some authors consider HS as a harbinger of withdrawal symptoms [19].

Despite the significant damage to health and large economic losses associated with HS, the pathogenesis of this condition is not well understood. At the same time, understanding the pathogenic mechanisms of HS is necessary for the development of means for the prevention and treatment of this condition. Presumptive mechanisms of HS pathogenesis are: Alcohol-induced metabolic and endocrine disorders, oxidative stress, inflammation, disturbances in water-electrolyte balance and acid-base balance [7,8,20-23].

The severity of HS depends on a number of factors, one of which is the dose of alcohol taken the night before [5]. The important role of the dose of alcohol consumed as a predictor of HS severity is confirmed by the fact that its severity correlates with the maximum alcohol concentration in the exhaled air, as well as with the concentration of alcohol and methanol in urine [8]. It should be noted that HS develops in the absence of alcohol in the blood. One of the factors influencing the severity of HS is the ethanol metabolic rate. It was found that in individuals with a slow ethanol metabolism and, accordingly, with a high level of alcohol in the urine, HS is more pronounced than in individuals with a fast ethanol metabolism [22].

An intriguing phenomenon in terms of understanding the pathogenic mechanisms of HS is resistance to its development. It is known that about $23 \%$ of subjects do not develop HS even after drinking a large dose of alco- hol [6]. The biological hypothesis suggests the existence of genetically determined differences in ethanol metabolism in persons experiencing HS and those resistant to it [24]. Twin studies have shown that the probability of occurrence of HS by $55.4 \%$ is determined by genetic factors [3]. It was found that the concentration of alcohol in the urine in the morning after alcoholic excess in persons with severe HS was significantly higher than in persons resistant to HS [5]. At the same time, the concentration of alcohol in the urine correlates with both the overall severity of $\mathrm{HS}$ and its individual symptoms [6]. Differences in the concentration of alcohol in the urine in persons with severe HS and those resistant to it indicate an important role of the ethanol metabolic rate in the pathogenesis of HS.

It has also been shown that HS resistance correlates with low sensitivity to the acute effects of alcohol [8]. It is known that low sensitivity to the acute effects of alcohol is a predictor of the development of alcohol dependence [25]. It was also found that resistance to HS is associated with the so-called festival style of alcohol consumption (drinking large doses of alcohol for a short period of time) [10]. The constellation of these factors suggests that persons resistant to the development of HS have a higher risk of developing alcohol dependence.

According to the psychological hypothesis, ethanol metabolism is the same in subjects with and without $\mathrm{HS}$; however, subjects resistant to HS are less sensitive to its symptoms [8]. One of the studies showed no differences in demographic, psychological and a number of biological parameters (methanol, ethyl glucuronide, ethyl sulfate levels, proinflammatory cytokine levels, sensitivity to acute effects of ethanol) in persons with and without HS [26]. At the same time, it was found that people with borderline personality disorders are more likely to experience hangovers compared to the general population [5].

Alcohol has a hypoglycemic effect [26], which can cause the appearance of such symptoms of HS as fatigue, impaired attention, and headache. At the same time, the administration of glucose against the background of HS had no effect on the severity of its symptoms [27]. Alcohol-induced sleep disturbance may be a possible cause of cognitive decline the morning after a binge [1]. However, it was found that sleep disturbance does not correlate with the severity of HS [5]. The severity of HS symptoms largely depends on the effectiveness of sleep [6].

Changes in the composition of the intestinal microflora may play an important role in the pathogenesis of hangover syndrome [8]. It has been shown that chronic alcohol consumption leads to dysbiosis [7]. Alcohol-induced dysbiosis is characterized by an increase in the number of pro-inflammatory and a decrease in the number of anti-inflammatory bacteria [26]. In addi- 
tion, by damaging the intestinal wall, alcohol increases the permeability of the intestinal barrier, which leads to increased absorption of toxic products [3]. The combined effect of dysbiosis and damage to the intestinal barrier is endotoxemia and an increase in the level of proinflammatory cytokines TNF-alfa and IL-6 [20]. It is assumed that anti-inflammatory cytokines of intestinal origin cause inflammation in the brain, which explains the symptoms of hangover from the central nervous system [5].

A number of studies have presented data indicating the involvement of the immune system in the pathogenesis of HS $[1,20,26]$. In particular, it was found that the level of cytokines (IL-10, IL-12, IFN- $\gamma$ ) in the blood significantly increases 13 hours after drinking alcohol [8]. In another study, it was shown that the peak level of pro-inflammatory cytokines (IL-1, IL-2, IL-6, IL-8, IFN- $\gamma$, TNF- $\alpha$ ) in saliva is observed 6 hours after the last dose of alcohol, and then, within the next 2-3 hours returns to normal [20].

The severity of the symptoms of HS may depend on the type of alcoholic drink consumed. Some alcoholic beverages contain a large amount of toxic impurities (methanol, fusel oils, higher alcohols, aldehydes), which can increase the severity of HS. It was found that the use of alcoholic beverages with a high content of impurities (bourbon, whiskey, tequila) is accompanied by a more severe HS than the use of beverages with a low content of impurities $[28,29]$. However, these data do not cast doubt on the fact that alcohol is the main etiological factor of HS. Additional pathogenic mechanisms of HS can be dehydration (alcohol increases diuresis), impaired mineral metabolism (a decrease in the level of calcium and magnesium in the blood plasma), alcoholic lactate and ketoacidosis (due to increased formation of acetoacetate and beta-hydroxybutyric acid) [7,8,20-23].

Insufficient understanding of the pathogenetic mechanisms of HS complicates the development of scientifically proven effective means of its prevention and treatment. Nevertheless, at present, active work in this direction is being carried out in many countries of the world. The most promising approach to the development of methods for the prevention and relief of HS is the use of drugs that accelerate the metabolism of ethanol. Rapid elimination of ethanol and acetaldehyde from the body can reduce the severity of hangover [3032].

An important area of research is the study of the effect of various nutrients on the severity of HS. It is known that food intake (especially fatty food) on the eve or during alcohol consumption slows down its absorption and reduces the peak blood concentration, thereby weakening the manifestation of HS symptoms [33]. Some vitamins (nicotinic acid, B vitamins, vitamin C) and trace elements (selenium, zinc, copper, magne- sium, iron, vanadium), having a direct effect on alcohol metabolism, or through their antioxidant and anti-inflammatory properties, can affect the likelihood and severity manifestations of HS [34,35]. In particular, nicotinic acid (vitamin PP) in the body is converted to nicotinamide, which is part of NAD and NADP, which are involved in ethanol metabolism [26]. In a study on volunteers who do not suffer from alcohol dependence, it was shown that the use of nicotinic acid in the diet is negatively associated with the severity of the clinical symptoms of HS [32].

As for other vitamins, the data on their use for the relief of HS manifestations are rather contradictory. For example, Kahn, et al. found that oral use of vitamin B6 significantly reduced the severity of HS symptoms [36]. At the same time, Kelly, et al. showed that intravenous administration of a complex of $B$ vitamins and vitamin $C$ does not affect ethanol metabolism [34]. Laas conducted a double-blind, placebo-controlled study to investigate the effectiveness of the Morning Fit hangover remedy, which contains dry yeast and vitamins B1, B2 and B6 [37]. It was found that this agent does not reduce the level of alcohol and acetaldehyde in the blood and does not affect the severity of symptoms of PS [37]. The literature data on the use of microelements for the relief of $\mathrm{HS}$ are limited. In one of the few studies devoted to this problem, it was shown that the use of zinc in the diet reduces the severity of symptoms of HS [35].

Taking into account the increase in the level of proinflammatory cytokines after the consumption of alcohol in a large dose [20], a potentially promising way to stop PS is the use of drugs that reduce their level. Probiotics normalize the intestinal microflora, reduce the permeability of the intestinal wall, and reduce the production of proinflammatory cytokines [32]. It has been found that probiotics reduce the severity of inflammation in the nervous tissue caused by alcohol-induced endotoxemia [31]. It was shown that after a 3-month course of treatment with probiotics, alcohol abusers showed a decrease in the level of pro-inflammatory cytokines in the blood, and the symptoms of a hangover almost completely disappeared [31].

In the USA, a pilot clinical trial of the hangover drug JMI-001, which is a combination of a non-steroidal anti-inflammatory drug and an $\mathrm{H} 1$ receptor antagonist, was carried out [23]. It has been shown that taking this drug on the eve of alcohol consumption significantly reduces the severity of HS. In a recent study, it was found that intravenous administration of the peptide hormone ghrelin, produced by cells of the gastric mucosa, significantly reduces the severity of HS [38].

There is information in the literature on the effectiveness of using plant extracts for the relief of HS. In particular, Bang, et al., showed that Acanthopanax senticosus extract normalizes glucose levels, lowers C-re- 
active protein levels, and also reduces the severity of HS symptoms such as fatigue, dizziness, and headache [39]. Kim, et al. found that the extract from the fruit of Hovenia dulcis, containing heteropolysaccharides and dihydromyricitin, significantly reduces the level of proinflammatory cytokines in the blood plasma and reduces the severity of hangover symptoms [40]. In a double-blind, placebo-controlled study, Opuncia ficus indica extract was shown to reduce C-reactive protein levels, increase heat shock protein synthesis, halve the likelihood of developing severe HS, and significantly reduce the severity of HS symptoms [41]. Another study showed that a blend of polyphenols from Viscum album, Lycium chinense, Inonotus obliquus, and Acanthopanax senticosus significantly reduced blood alcohol content two hours after drinking [42].

In conclusion, the heavy burden of medical and social problems and economic damage associated with PS necessitates the development of measures for the prevention and treatment of this pathological condition. A review of the literature data showed the existence of significant gaps in understanding the pathogenesis of HS, which complicates the development of means of metabolic correction of this condition. The target of most of the currently proposed treatments for HS is any one of the pathogenic mechanisms of HS. The effectiveness of most of the available drugs has not been confirmed by clinical trials. In addition, many of them have side effects that limit their use. Therefore, an urgent task for further research is a detailed study of the pathogenic mechanisms of HS, which will make it possible to develop a comprehensive treatment for HS that affects all links of its pathogenesis. An independent direction of research can be the development of means for the prevention of HS. It should be understood that the best way to prevent HS is to stop drinking alcohol.

\section{Funding}

The study had no sponsorship.

\section{Conflict of Interests}

The authors declare no conflict of interests.

\section{References}

1. Razvodovsky YE (2012) Alcoholism: medical and social aspects - Saarbrucken: LAP Lambert Academic Publishing Gmb \& Co. KG. 294.

2. Nuznyj VP, Tesicov EB, Uspensky AE (1995) Postintoxication alcohol syndrome. The Questions of Narcology 2: 50-59.

3. Prat G, Adan A, Sánchez-Turet M (2009) Alcohol hangover: A critical review of explanatory factors. Hum Psychopharmacol 24: 259-267.

4. Van Schrojenstein Lantman M, van de Loo AJ, Mackus M, Verster JC (2016) Development of a definition for the alcohol hangover: Consumer descriptions and expert consensus. Curr Drug Abuse Rev 9: 148-154.

5. Wiese JG, Shlipak MG, Browner WS (2000) The alcohol hangover. Ann Intern Med 132: 897-902.
6. Becker J (2001) The alcohol hangover. Ann Intern Med 134: 533-534.

7. Palmer E, Tyacke R, Sastre M, Lingford-Hughes $A$, Nutt D, et al. (2019) Alcohol Hangover: Underlying Biochemical, Inflammatory and Neurochemical Mechanisms. Alcohol Alcohol 54: 196-203.

8. Penning R, van Nuland M, Fliervoet LAL, Olivier B, Verster JC (2010) The pathology of alcohol hangover. Current Drug Abuse Revew 3: 68-75.

9. Streufert S, Pogash R, Braig D, Gingrich D, Kantner A, et al. (1995) Alcohol hangover and managerial effectiveness. Alcohol Clin Exp Res 19: 1141-1146.

10. Swift S, Davidson D (1998) Alcohol hangover: Mechanisms and mediators. Alcohol Health Res World 22: 54-60.

11. Kauhanen J, Kaplan GA, Goldberg DD, Cohen RD, Lakka TA, et al. (1997) Frequent hangovers and cardiovascular mortality in middle-aged men. Epidemiology 8: 310-314.

12. Kim DJ, Yoon SJ, Lee HP, Choi BM, Go HJ (2003) The effects of alcohol hangover on cognitive functions in healthy subjects. Int J Neurosci 113: 581-594.

13. Lemon J, Chesher G, Fox A, Greeley J, Nabke C (1993) Investigation of the "hangover" effects of an acute dose of alcohol on psychomotor performance. Alcohol Clin Exp Res 17: 665-668.

14. Scholey A, Benson S, Kaufman J, Terpstra C, Ayre E, et al. (2019) Effects of alcohol hangover on cognitive performance: A field/internet mixed methodology approach. J Clin Med 8: 440.

15. Seppala T, Leino T, Linnoila M, Huttunen M, Ylikahri $R$ (1976) Effects of hangover on psychomotor skills related to driving: Modification by fructose and glucose. Acta Pharmacol Toxicol Copenh 38: 209-218.

16. Verster JC, van Duin D, Volkerts ER, Schreuder AHCML, Verbaten MN (2003) Alcohol hangover effects on memory functioning and vigilance performance after an evening of binge drinking. Neuropsychopharmacology 28: 740-746.

17. Tornros J, Laurell H (1991) Acute and hang-over effects of alcohol on simulated driving performance. Blutalkohol 28 : 24-30.

18. Razvodovsky YE (2013) Narcology- Saarbrucken: LAP Lambert Academic Publishing 137.

19. Wang F, Li Y, Zhang YJ, Zhou Y, Li S, et al. (2016) Natural Products for the Prevention and Treatment of Hangover and Alcohol Use Disorder. Molecules 21: 64.

20. Kim DJ, Kim W, Yoon SJ, Choi BM, Kim JS, et al. (2003) Effects of alcohol hangover on cytokine production in healthy subjects. Alcohol 31: 167-170.

21. Mackus $M$, van Schrojenstein Lantman $M$, Van de Loo AJAE, Kraneveld AD, Garssen J, et al. (2018) Alcohol metabolism in hangover sensitive versus hangover resistant social drinkers. Drug Alcohol Dependence 185: 351-355.

22. Tipple CT, Benson S, Scholey A (2016) A Review of the Physiological Factors Associated with Alcohol Hangover. Curr Drug Abuse Rev 9: 93-98.

23. Verster JC, Vermeulen SA, Van de Loo AJAE, Balikji S, Kraneveld AD, et al. (2019) Dietary Nutrient Intake, Alcohol Metabolism and Hangover Severity. J Clin Med 8: 1316.

24. Bouwmeester NH, Brookhuis KA, Garssen J, Verster JC (2016) Characteristics of social drinkers with and without a hangover after heavy alcohol consumption. Subst Abuse Rehabil 7: 161-167. 
25. Ostrovsky YM, Satanovskaya VI, Sadovnic MN (1986) Biological component in the genesis of alcoholism. Minsk 95.

26. Harburg E, Gunn R, Gleiberman LD, Franceisco W, Schork A (1993) Psychosocial factors, alcohol use, and hangover signs among social drinkers: A reappraisal. J Clin Epidemiolo 46: 413-422.

27. Ylikahri RH, Leino T, Huttunen MO, Pösõ AR, Eriksson CJP, et al. (1976) Effects of fructose and glucose on ethanol-induced metabolic changes and on the intensity of alcohol intoxication and hangover. Eur J Clin Invest 6: 93-102.

28. Damrau F, Liddy E (1960) Hangovers and whisky congeners: Comparison of whisky with vodka. J Natl Med Assoc 52: $262-265$

29. Rohsenow DJ, Howland J (2010) The role of beverage congeners in hangover and other residual effects of alcohol intoxication: A review. Curr Drug Abuse Rev 3: 76-79.

30. Mackus $M$, van Schrojenstein Lantman $M$, van de Loo AJAE, Nutt DJ, Verster JC (2017) An effective hangover treatment: Friend or foe? Drug Science Policy Law 3: 1-7.

31. Pittler MH, Verster JC, Ernst E (2005) Interventions for preventing or treating alcohol hangover: Systematic review of randomised controlled trials. BMG 331: 1515-1518.

32. Verster JC, Penning R (2010) Treatment and prevention of alcohol hangover. Curr Drug Abuse Rev 3: 103-109.

33. Finnigan F, Hammersley R, Millar KE (1998) Effects of meal composition on blood alcohol level, psychomotor performance and subjective state after ingestion of alcohol. Appetite 31: 361-375.

34. Kelly M, Myrsten AL, Goldberg L (1971) Intravenous vitamins in acute intoxication: Effects on physiological and psychological functions. $\mathrm{Br} \mathrm{J}$ Addict Alcohol other Drugs 66: 19-30.
35. Min JA, Lee K, Ki DJ (2010) The application of minerals in managing alcohol hangover: A preliminary review. Curr Drug Abuse Rev 3: 110-115.

36. Kahn MA, Jensen K, Krogh HJ (1973) Alcohol-induced hangover. A double-blind comparison of pyritinol and placebo in preventing hangover symptoms. Q J Stud Alcohol 34: 1195-1201.

37. Laas IA (1999) Double-blind placebo-controlled study on the effects of Morning Fit on hangover symptoms after a high level of alcohol consumption in healthy volunteers. Journal Clinical Research 2: 9-15.

38. Farokhnia M, Lee MR, Farinelli LA, Ramchandani VA, Akhlaghi $F$, et al. (2018) Pharmacological manipulation of the ghrelin system and alcohol hangover symptoms in heavy drinking individuals: Is there a link? Pharmacol Biochem Behav 172: 39-49.

39. Bang JS, Chung YH, Chung SJ, Lee HS, Song EH, et al. (2015) Clinical effect of a polysaccharide-rich extract of Acanthopanax senticosus on alcohol hangover. Pharmazie 70: $269-273$.

40. Kim H, Kim YJ, Jeong HY, Kim JY, Choi EK, et al. (2017) A standardized extract of the fruit of Hovenia dulcis alleviated alcohol-induced hangover in healthy subjects with heterozygous ALDH2: A randomized, controlled, crossover trial. J Ethnopharmacol 209: 167-174.

41. Wiese J, McPherson S, Odden MC, Shlipak MG (2004) Effect of Opuntia ficus indica on symptoms of the alcohol hangover. Arch Intern Med 164: 1334-1340.

42. Hogewoning $A$, van de Loo AJAE, Mackus $M$, Raasveld SJ, de Zeeuw R, et al. (2016) Effects of the herb mixture, DTS20, on oxidative stress and plasma alcoholic metabolites after alcohol consumption in healthy young men. Integr Med Res 5: 309-316. 